\title{
Is There a Relationship between the Functional Reach Test and Flexibility?
}

\author{
Masanobu Uchiyama ${ }^{1}$, Shinichi Demura ${ }^{2}$, Sohee Shin $^{3}$ \\ ${ }^{1}$ Research and Education Center for Comprehensive Science, Akita Prefectural University, Akita, Japan; \\ ${ }^{2}$ Graduate School of Natural Science and Technology, Kanazawa University, Kanazawa, Japan; \\ ${ }^{3}$ Center for Innovation Venture Business Laboratory, Kanazawa University, Kanazawa, Japan. \\ Email: masanobu.uchiyama@gmail.com \\ Received August 28 $8^{\text {th }}, 2011$; revised September $30^{\text {th }}, 2011$; accepted October $10^{\text {th }}, 2011$.
}

\begin{abstract}
This study examined the influence of short-term stretching and improved flexibility on functional reach (FR) performances (reach distance and rotation angle of various joints during reaching). 17 healthy male university students were assigned to either experimental and control groups based on the random assignment. A pre-test/post-test design was used in this study. The variable factor for the experimental group was stretching (jogging for warming up and stretching). Main outcome measures were flexibility (static maximum range of motion of shoulder, hip, and ankle joints) and FR of participants. FR test performances were assessed by the reach distance and rotation angles of each joint during reaching. No significant differences between the groups were found in the participants' characteristics, and no skeletal muscle fatigue was found in the lower limb. In the experimental group, shoulder and hip-joint flexibility increased significantly by stretching, and showed higher values than that of the control group. In contrast, FR test performance did not reveal any changes. Slight intraindividual ROM improvements by short-term stretching may be less important for FR postural strategies. FR test performances are little influenced by light static stretching in young healthy adults.
\end{abstract}

Keywords: Evaluation, Postural Balance, Flexibility, Reach

\section{Introduction}

Humans maintain an upright stance by combining vestibular, visual and somatosensory information (Fransson, Kristinsdottri, Hafstrom, Magnusson, \& Johansson, 2004; Vuillerme, Pinsault, $\&$ Vaillant, 2005). These various functions contribute to balancing ability, which can be assessed by the functional reach test (FRT) (Duncan, Weiner, Chandle, \& Studenski, 1990) and single-limb stance test (Zumbrunn, Macwilliams, \& Johnson, 2011), among others. The FRT was developed essentially to minimize fall risk for the elderly. However, it is now being used for all age groups.

The functional reach test (FRT) (Duncan et al., 1990) is a simple test for balance assessment in which the maximal forward reach distance is measured. FRT scores are useful for screening fall risk of the elderly with a cutoff point subtracted $15 \mathrm{~cm}$ from a standard value as well as for balance assessment (Duncan et al., 1990). It was reported that the FRT has good intra-rater and inter-rater reliabilities and correlates with gait velocity, tandem gait, and single-limb stance (Whitney, Poole, \& Cass, 1998).

On the other hand, Nakamura et al. (2006) referred to many previous studies that question the validity of the FRT as a performance balance measure (Fujisawa, Takeda, Maeda, \& Hayakawa, 2005; Takakura \& Ohgi, 2005; Thomas \& Lane, 2005; Tsushima, Tsushima, Ishida, Hasegawa, \& Ohkura, 2001; Wernick-Robinson, Krebs, \& Giogetti, 1999). Wernick-Robinson et al. (1999) reported that there was no difference in the FRT scores between the healthy elderly and the elderly with balance disorders. Similarly, Yamada and Ichihashi (2010) reported no significant score differences for both groups. Fujisawa et al. (2005) also reported that the FRT showed no significant correlations with the Timed "Up \& Go", the $10 \mathrm{~m}$ walk in the elderly and the single-limb stance with closed eyes, and the body sway path length in young adults.

Recently, many studies have suggested correlations between the FRT and flexibility, or the range of joint motion (Nakamura et al., 2006; Takakura \& Ohgi, 2005). For example, Thomas and Lane (2005) reported that the FRT reflects the flexibility of the trunk rather than movement of the center of mass (COM); therefore, it cannot be used as a real balance measure. However, the relationship between the FR test performance and flexibility has been little clarified. Examining this relationship may be important for understanding the validity of the FR test as a balance measure.

In this study, we hypothesized that the FR test score would improve with short-term stretching and increased joint flexibility.

\section{Methods}

\section{Participants}

Seventeen healthy university students of age $(21.9 \pm 1.2)$ years, height $(172.8 \pm 5.2) \mathrm{cm}$ and body mass $(68.6 \pm 6.6) \mathrm{kg}$ participated in this study, values are mean \pm SD. Their physical characteristics were almost the same as the age-matched national standard value (Laboratory of Physical Education Tokyo Metropolitan University, 2000). Participants were assigned to experimental $(\mathrm{n}=7$; age $=(21.9 \pm 1.5) \mathrm{yrs}$; height $=(171.7 \pm$ $6.5) \mathrm{cm}$; body mass $=(69.7 \pm 8.1) \mathrm{kg})$ and control $(\mathrm{n}=10$; age $=(22.0 \pm 1.2) \mathrm{yrs}$; height $=(172.7 \pm 4.7) \mathrm{cm}$; body mass $=(67.8$ $\pm 6.1) \mathrm{kg}$ ) groups based on the random assignment. Prior to the measurements, the purpose and procedures of this study were explained in detail to all participants and written informed consent was obtained. This experimental protocol was approved by 
the ethics committee (Kanazawa University Health and Science Ethics committee).

\section{Experimental Condition}

This study used the classic controlled experimental design, i.e. randomized pre-test/post-test design. Namely, the participants were randomly assigned to two groups or an experimental group which is subjected to a variable factor (in this case, stretching) and a control group which is not subjected to the factor. The variable consisted of stretching exercises used to improve flexibility (jogging and static stretch). It has been reported that an active and static stretch increases the flexibility of muscle fibers and tendons (Safran, Garrett, Seaber, Glisson, \& Ribbeck, 1988).

All participants were instructed not to sleep, exercise intently, or eat for 2 hours before this experiment. First, the participants sat quietly on a chair for $10 \mathrm{~min}$. The room temperature was kept at 23 degrees C. An experienced tester measured physical characteristics (height and body mass) and resting heart rate (HR) of each participant in both groups. Then base line measurements of muscle strength, flexibility (ROM of shoulder, hip and ankle joints), and FR test were conducted. Following these initial measurements, the intervention was carried out only for the experimental group. Lastly, muscle strength, flexibility, and FR test results were measured for both groups, again.

The stretching consisted of the following; first, jogging for $15 \mathrm{~min}$ at $60 \% \mathrm{HR}$ reserve was done followed by an active static stretch for neck, shoulder, hip and ankle joints for $5 \mathrm{~min}$ (30 sec per each joint). The heart rate during the warm-up jogging was monitored by an electrocardiographic monitor (Aero Cardinar, Minato Ikagaku, Japan). A tester indicated the pace of jogging to make each participant keep a certain heart rate.

\section{Measurement Items}

\section{Functional Reach}

In the FRT, the maximum forward reaching distance of participants was measured during standing on both feet and while fixing their base of support (Duncan et al., 1990). Prior to the FRT, participants stood in a comfortable position in parallel with a rope stretched at their shoulder height on their sagittal plane. They then flexed their shoulder 90 degrees by extending their arm and closing their fist. A tester marked the front edge position of each participant's third metacarpal bone on the above-stated stretched rope. Next, participants reached forward as far as they possibly could without losing their balance. A tester recorded the distance between the first marked position of their third metacarpal bone and the position of the bone when participants reached maximally. The participants were instructed to reach with their dominant arm. All participants were right handed.

\section{Flexibility}

The maximum active flexion and extension angles of the shoulder, hip and ankle joints without any assistance were measured using a regular goniometer (Yagami, Japan). Flexibility measurements were conducted by a well practiced tester. Measurements were conducted twice for each joint after one practice trial, and the average of the two trials was used for further analysis.

\section{Strength of Plantar Flexion}

In the FR test, ankle and hip postural strategies were used in conjunction with a body trunk rotation. Plantar flexors of the lower limbs are involved in the ankle strategy. To confirm that there was no plantar flexor muscle fatigue (a decrease in muscle strength) from intervening stimuli, maximum plantar flexion

\begin{tabular}{|c|c|c|c|}
\hline \multicolumn{4}{|c|}{ 1. Protocol for the experimental group } \\
\hline Rest & Pre-test & Stretching & Post-test \\
\hline (for $10 \mathrm{~min}$ ) & (for $30 \mathrm{~min}$ ) & $\begin{array}{l}\quad \text { (for } 20 \mathrm{~min} \text { ) } \\
\text { (Jogging at } 60 \% \text { Hrreserve } \\
\text { and Stretchingof neck, } \\
\text { shoulder, hip and ankle } \\
\text { joints) }\end{array}$ & (for $30 \mathrm{~min}$ ) \\
\hline Rest & Pre-test & Rest & Post-test \\
\hline (for $10 \mathrm{~min}$ ) & (for $30 \mathrm{~min}$ ) & (for $20 \mathrm{~min}$ ) & (for $30 \mathrm{~min}$ ) \\
\hline
\end{tabular}

Figure 1.

Experimental protocols.

Table 1.

Measurement items in each experimental phase.

\begin{tabular}{|c|c|}
\hline Phase & Item \\
\hline & -Physique \\
\hline & - Resting heart rate \\
\hline \multirow[t]{3}{*}{ Pre-test phase } & -Flexibility measures (ankle, hip, shoulder joint angles) \\
\hline & - Maximal plantarflexion strength \\
\hline & -Functional reach \\
\hline \multirow[t]{3}{*}{ Post-test phase } & -Flexibility measures (ankle, hip, shoulder joint angles) \\
\hline & - Maximal plantarflexion strength \\
\hline & - Functional reach \\
\hline
\end{tabular}


muscle strength was measured before (pre-test) and after (post-test) the intervention, using a handheld dynamometer ( $\mu$-Tas, ANIMA, Japan). The participants were instructed to sit down on the floor of the experimental room with extended legs, and to keep the ankle joints at 90 degrees. Then, with maximal effort, they used the hallux (big toe) joint of their dominant foot to push the small force-plate of a handheld dynamometer, which was affixed to a wall at the height corresponding to their hallus joint.

\section{Apparatus}

\section{Motion Analysis System}

The rotation angles of several of the participants' joints during the FRT was recorded and analyzed by a motion analysis system (MA-200X, ANIMA, Japan). This system can record each participant's reaching motion from either side and from diagonally forward or backward using a six video camera setup.

A tester attached 16 infrared-light emitting markers on the participant's anatomical landmarks and recorded a dynamic image of their reaching motion with a sampling frequency of 60 $\mathrm{Hz}$. The markers were placed bilaterally over the acrominon, cubitus, carpus, iliac crest, great trochanter, knee joint, ankle joint and metatarsal bone. The six cameras were set at $3 \mathrm{~m}$ forward and $3 \mathrm{~m}$ backward diagonally, $0 \mathrm{~m}$ (right and left), with 6 $\mathrm{m}$ apart from the participant's sagittal plane.

\section{Handheld dynamometer}

A handheld dynamometer $\mu$-Tas (ANIMA, Japan) was used to measure a maximum plantarflexion strength before and after a warm-up and stretching intervention. The $\mu$-Tas consists of a sensor and a display. A small force plate was used as a sensor. Its pressure receiver and sensor are united together, thus it can measure with high accuracy.

\section{Parameters}

Maximum flexion and extension angles and static maximum range of motion, i.e. sum of the flexion and extension angles, of the shoulder, hip, and ankle joints were calculated as flexibility measures.

To assess the reaching motion in a FRT, the difference between the joint angle at maximum reaching posture and the angle at preparation posture was calculated for the shoulder joint, hip joint and dorsiflexion angles. The maximum reaching posture means the posture in which the participants reached as far forward as possible. The preparation posture means the posture when they extended the reaching arm prior to the onset of the FRT and flexed the shoulder joint at 90 degrees.

\section{Data Analysis}

The mean differences of the participants' characteristics be- tween both groups were examined by an unpaired t-test. To examine the influence of the intervention stimuli on the participants' flexibility, lower limb muscle strength and FRT performance, a two-way ANOVA which tested the group factor (experimental vs. control) and the test with repeated measures (pre- and post-tests) was used. When significant interaction effect occurred, Tukey's honestly significant difference (HSD) post-hoc test was used. A value of $\mathrm{p}<0.01$ was considered to be significant.

\section{Results}

Table 2 shows the results of the unpaired t-test for participants' characteristics. No significant difference between groups was found in all items. Therefore, both groups were considered to be identical. Table 3 shows the results of ANOVA and post-hoc test for parameters of flexibility (ROM), lower leg muscle strength and FRT performances. Significant interaction effect was found in ROM for shoulder and hip joints. These parameters were significantly higher after the intervention in the experimental group and were significantly higher in the experimental group than in the control group after the intervention. No treatment effect was found in lower limb muscle strength and FRT performances. Briefly, muscle fatigue in the lower limb did not arise due to this intervention for flexibility improvement. In discussion, the reasons why the improvement in mobility of various joints has no influence on the FRT performances were described.

\section{Discussion}

The functional reach test has been commonly used as a clinical test measuring the limit of stability (margin of stability) and was reported to have high reliability and validity as a balance test (Duncan et al., 1990). However, participant's flexibility, particularly range of motion (ROM) of the shoulder and/or hip joints, may largely influence the reaching distance, because the motion task of this test requires maximal extension of the extremities and a large flexing action of the hip and shoulder joints. In this study, the FRT was conducted before and after a short-term intervention that increased joint mobility.

First, it was confirmed that there was no decline in maximal plantar flexion strength due to the intervention and the range of motion of shoulder and hip joints significantly increased. In this study, moderate exercise of $60 \%$ HR reserve and static stretching exercise were selected as the intervention to increase the participants' ROM. This intervention was judged to be valid due to increasing flexibility of all the body joints including the shoulder, hip, and ankle joints without muscle fatigue of lower limbs.

Table 2.

Differences of physical characteristics between control and experimental groups.

\begin{tabular}{|c|c|c|c|c|c|c|}
\hline & & $\mathrm{EG}(\mathrm{n}=7)$ & $\mathrm{CG}(\mathrm{n}=10)$ & & & \\
\hline & & Mean \pm S.D. & Mean \pm S.D. & t-value & $\mathrm{p}$-value & \\
\hline Age & (yrs) & $21.9 \pm 1.46$ & $22.0 \pm 1.15$ & -0.23 & .82 & n.s. \\
\hline Heigh & $(\mathrm{cm})$ & $171.7 \pm 6.47$ & $172.7 \pm 4.70$ & -0.35 & .73 & n.s. \\
\hline \multirow[t]{2}{*}{ Weight } & $(\mathrm{kg})$ & $69.7 \pm 8.10$ & $67.8 \pm 6.08$ & 0.57 & .58 & n.s. \\
\hline & & s.: Not significa & perimental gro & ol group & & \\
\hline
\end{tabular}


Table 3.

The Mean differences in flexibility (ROM), lower leg muscle strength and FRT performances between both groups.

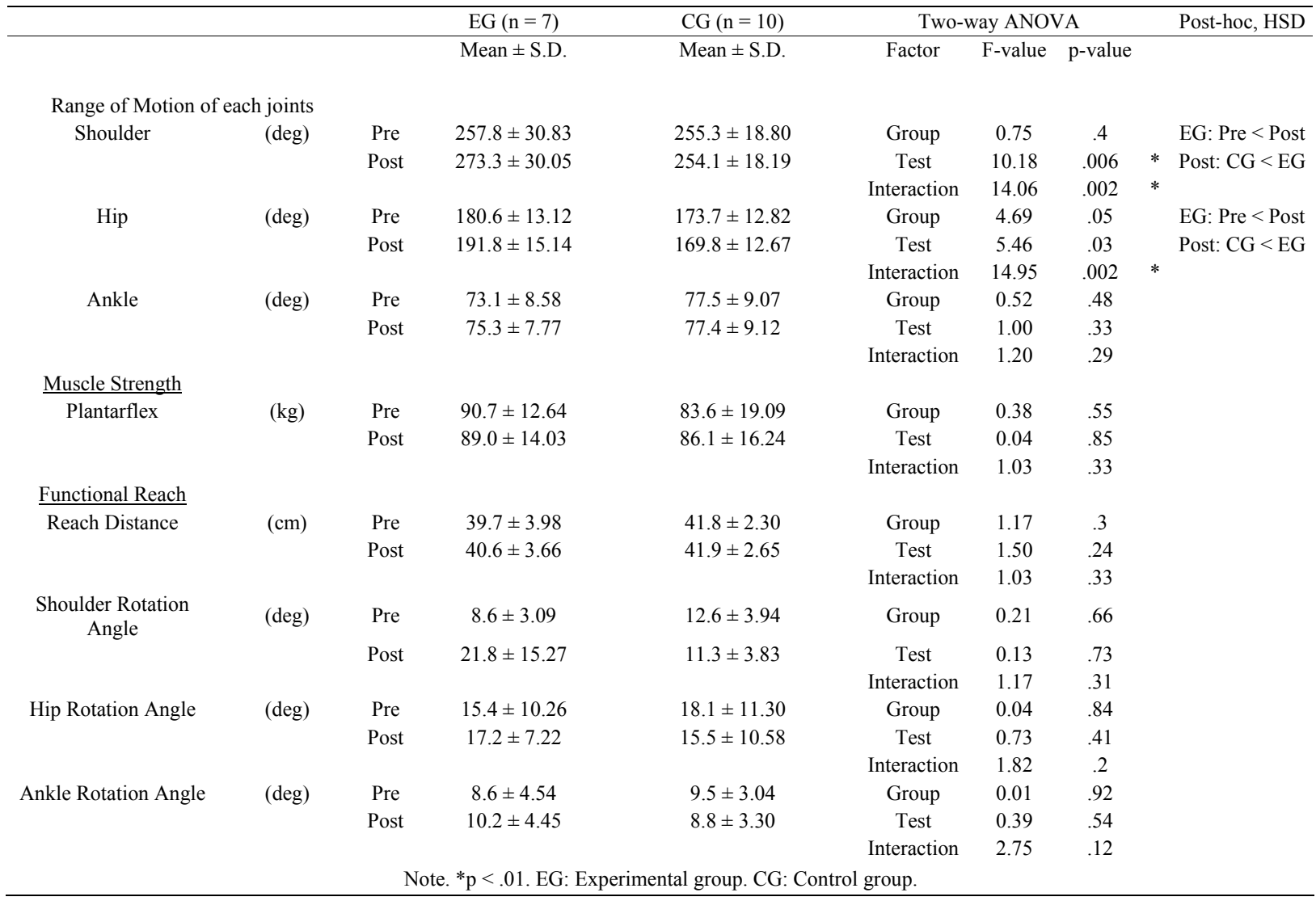

In contrast, there was no intervention effect in the FRT per formances (reach distance and rotation angle of various joints during reaching). The stretching used in this study increased the ROM of various joints. However, it was suggested that this change in ROM makes neither the motion nor the reach distance of the FRT change. This finding disproved our hypothesis. Barrett and Smerdely (2002) used elderly participants to examine the influences of long-term (10 months) progressive resistant training routines and flexibility exercises on FRT scores. They reported that the FRT improvement ratio of the incremental strength training group was $11.7 \%$ larger than that of the flexibility exercise group. The intervention time period and demographic of the present study are different from those used by Barrett and Smerdely. However, both studies suggest that long- and short-term stretching exercises and subsequent intraindividual improvements in mobility of various joints have no influence on the FRT score.

Although healthy young adults were tested in this study, it will be important to assess balance ability in the elderly with a high risk of falling using a simple performance balance measure, e.g. the FRT. Various elements of physical fitness in the elderly clearly decreases compared with young adults. The elderly must perform various activities of daily living with decreased physical fitness. Mecagni, Smith, Roberts, O'sullivan (2004) examined the relationship between the ankle range of motion (ROM), which has been reported to decrease with aging, and the FRT score using elderly females, and observed a significant correlation between them. Further research considering the age level difference in physical fitness elements between age levels will be needed.

\section{Conclusions}

In conclusion, in healthy young adults, improvement of the range of motion of joints by warming-up and stretching has little influence on FRT scores.

\section{References}

Barrett C. J., \& Smerdely P. (2002). A comparison of community-based resistance exercise and flexibility exercise for seniors. The Australian Journal of Physiotherapy, 48, 215-219.

Boulgarides, L. K., McGinty, S. M., Willett, J. A., \& Barnes, C. W. (2003). Use of clinical and impairment-based tests to predict falls by community-dwelling older adults. Physical Therapy, 83, 328-339.

Duncan, P. W., Weiner, D. K., Chandle, J., \& Studenski, S. (1990). Functional reach: A new clinical measure of balance. Journal of Gerontology, 45, 192-197.

Fransson, P. A., Kristinsdottri, E. K., Hafstrom, A., Magnusson, M., \& Johansson, R. (2004). Balance control and adaptation during vibratory perturbations in middle-aged and elderly humans. European Journal of Applied Physiology, 91, 595-603. doi:10.1007/s00421-003-1013-1

Fujisawa, H., Takeda, R., Maeda, S., \& Hayakawa, Y. (2005). Significance of functional reach test and one-footed standing duration in hemiplegia: Relationship between balance and walking abilities. Journal of the Japanese Physical Therapy Association, 32, 416-422. [In Japanese with English Abstract]

Laboratory of Physical Education Tokyo Metropolitan University. (2000). New physical fitness standards of Japanese people (5th ed.). Tokyo: Fumaido. [In Japanese]

Mecagni, C., Smith, J. P., Roberts, K. E., O’Sullivan, S. B. (2000). Balance and ankle range of motion in community-dwelling women aged 64 to 87 years: A correlational study. Physical Therapy, 80, 
1004-1011.

Nakamura, I., Okuda, M., Kage, H., Kunitugu, I., Sugiyama, S., Hobara, T., \& Asami, I. (2006). The relationship between a functional reach test and other balance tests. Rigakuryoho Kagaku, 21, 335-339. [In Japanese with English Abstract] doi:10.1589/rika.21.335

Safran, M. R., Garrett, W. E. Jr., Seaber, A. V., Glisson, R. R., \& Ribbeck, B. M. (1988). The role of warmup in muscular injury prevention. The American Journal of Sports Medicine, 16, 123-129. doi: $10.1177 / 036354658801600206$

Takakura, S., \& Ohgi, S. (2005). The relationship between the standing postural control test using the elderly balance board type $\mathrm{N}$ and gait performance. Rigakuryoho Kagaku, 20, 315-319. [In Japanese with English Abstract] doi:10.1589/rika.20.315

Thomas, J. I., \& Lane, J. V. (2005). A pilot study to explore the predictive validity of 4 measures of falls risk in frail elderly patients. Archives of Physical Medicine and Rehabilitation, 86, 1636-1640. doi:10.1016/i.apmr.2005.03.004

Tsushima, E., Tsushima, H., Ishida, M., Hasegawa, T., \& Ohkuma, K. (2001). Correlation of functional reach distance, sagittal displacement and envelope area of the center of gravity in functional reach test by hip, ankle, and heels-up strategy in normal subjects. Riga- kuryoho Kagaku, 16, 159-165. [In Japanese with English Abstract] doi:10.1589/rika.16.159

Vuillerme, N., Pinsault, N., \& Vaillant, J. (2005). Postural control during quiet standing following cervical muscular fatigue: Effects of changes in sensory inputs. Neuroscience Letters, 378, 135-139. doi:10.1016/j.neulet.2004.12.024

Wernick-Robinson, M., Krebs, D. E., \& Giorgetti, M. M. (1999). Functional reach: Does it really measure dynamic balance? Archives of physical medicine and rehabilitation, 80, 262-269. doi:10.1016/S0003-9993(99)90136-3

Whitney, S. L., Poole, J. L., \& Cass, S. P. (1998). A review of balance instruments for older adults. The American journal of occupational therapy. Official Publication of the American Occupational Therapy Association, 52, 666-671. doi:10.5014/ajot.52.8.666

Yamada M., \& Ichihashi N. (2010). Predicting the probability of falls in community-dwelling elderly individuals using the trail-walking test. Environmental Health and Preventive Medicine, 15, 386-391.

doi:10.1007/s12199-010-0154-1

Zumbrunn T., Macwilliams B. A., \& Johnson B. A. (2011). Evaluation of a single leg stance balance test in children. Gait \& Posture, 34, 174-177. doi:10.1016/j.gaitpost.2011.04.005 